\title{
Aptamer-based molecular imaging
}

\author{
Tianjiao Wang $^{\bowtie}$, Judhajeet Ray \\ Department of Biochemistry, Biophysics and Molecular Biology, lowa State University, Ames, IA 50011, USA \\ $\triangle$ Correspondence: tjwang@iastate.edu \\ Received June 24, 2012 Accepted August 9, 2012
}

\begin{abstract}
Molecular imaging has greatly advanced basic biology and translational medicine through visualization and quantification of single/multiple molecular events temporally and spatially in a cellular context and in living organisms. Aptamers, short single-stranded nucleic acids selected in vitro to bind a broad range of target molecules avidly and specifically, are ideal molecular recognition elements for probe development in molecular imaging. This review summarizes the current state of aptamer-based biosensor development (probe design and imaging modalities) and their application in imaging small molecules, nucleic acids and proteins mostly in a cellular context with some animal studies. The article is concluded with a brief discussion on the perspective of aptamer-based molecular imaging.
\end{abstract}

KEYWORDS aptamer, imaging, small molecule, nucleic acid, protein, biosensor

\section{INTRODUCTION}

Molecular imaging is to visualize and quantify a molecular event temporally and spatially in a cellular context and in living organisms (Massoud and Gambhir, 2003; Weissleder and Pittet, 2008; Pysz et al., 2010). In basic biology, molecular imaging has been applied widely to study different molecular events including gene expression, molecular trafficking/localization and molecular interactions. In translational medicine, it has been used routinely in disease target validation, drug discovery and diagnosis. Molecular imaging is rapidly emerging as an interdisciplinary subject and has greatly advanced both basic biology and translational medicine.

In targeted molecular imaging, a biosensor is developed to specifically recognize and image a target molecule. The rec- ognition elements used in a biosensor can be either small molecules (e.g., metabolites, enzymatic substrates and neurotransmitters), antibodies or aptamers. In this review, we will focus only on molecular imaging using nucleic acid aptamers as recognition elements in biosensors.

Aptamers are short ssDNAs/RNAs (in the range of 15-150 $\mathrm{nt}$, ideally $20-40 \mathrm{nt})$ that bind their targets with high affinity $\left(\mathrm{K}_{\mathrm{d}}\right.$ : $\mu \mathrm{mol} / \mathrm{L}-\mathrm{pmol} / \mathrm{L}$ ) and specificity. Aptamers are selected in vitro by Systematic Evolution of Ligands by EXponential enrichment (SELEX), which was first established in 1990 (Ellington and Szostak, 1990; Tuerk and Gold, 1990). Aptamers have been selected for a broad range of targets including small molecules, carbohydrates, peptides/proteins, nucleic acids, lipids and complex targets including protein complexes, viral particles, cells, tissues and small living organisms (Wang, 2008). Aptamers are ideal probe reagents for biosensor development in molecular imaging with their properties of broad target range, high specificity and affinity, capability of in vitro chemical synthesis and modifications for improving functionality and pharmacokinetics (biostability with half-life of 1-10 days in serum with chemical modifications and circulation half-life of minutes without PEGylation and days with PEGylation) (Keefe et al., 2010), flexibility and often induced-fit in structural/conformational changes upon target binding, lack of immunogenicity and the capability of functioning both in vitro and in vivo.

Aptamers have great potential in therapy (Keefe et al., 2010), diagnosis (Brody et al., 2010), in vitro bioanalysis (Iliuk et al., 2011) and in vivo imaging (Hong et al., 2011; Lopez-Colon et al., 2011). The combination of aptamer with nanotechnology is also an actively studied field (Yang et al., 2011). In this review, we focus only on aptamer-based molecular imaging with a comprehensive summary of target molecules imaged. We first summarize the principles of aptamer-based biosensor development (probe design and imaging modality) in the context of molecular imaging. Then we describe the application of aptamer-based biosensors in imaging various molecular events including small molecules, 
nucleic acids and proteins.

\section{APTAMER-BASED BIOSENSOR DEVELOPMENT}

Aptamer-based biosensor development for in vitro bioanalysis has been thoroughly reviewed (Iliuk et al., 2011) and will not be covered here. Here, we describe the principle of aptamer-based biosensor development in the context of molecular imaging. An aptamer-based biosensor for imaging usually consists of an aptamer probe and an imaging modality. In the probe, there is a sensing domain and a signaling domain. The sensing domain first recognizes a target molecule by a recognition element (an aptamer). The recognition event is then transduced to a signaling domain to give out a signal through a reporter, which can be measured by an imaging modality. The reporters can be fluorophores, radionuclides, magnetic reagents, microbubbles and other contrast reagents (reagents for enhancing image contrast). Correspondingly, the imaging modalities can be fluorescence microscopy, radiography, magnetic resonance imaging (MRI), ultrasound imaging and computed tomography (CT) (Fig. 1). The aptamer-based biosensor development is discussed below, including the principles of aptamer probe design, different imaging modalities, signal amplification for imaging and multiplex imaging.

\section{Aptamer probe development}

Aptamer probe development is an essential part of aptamer-based molecular imaging. For successful molecular imaging, a probe should possess physical properties includ-

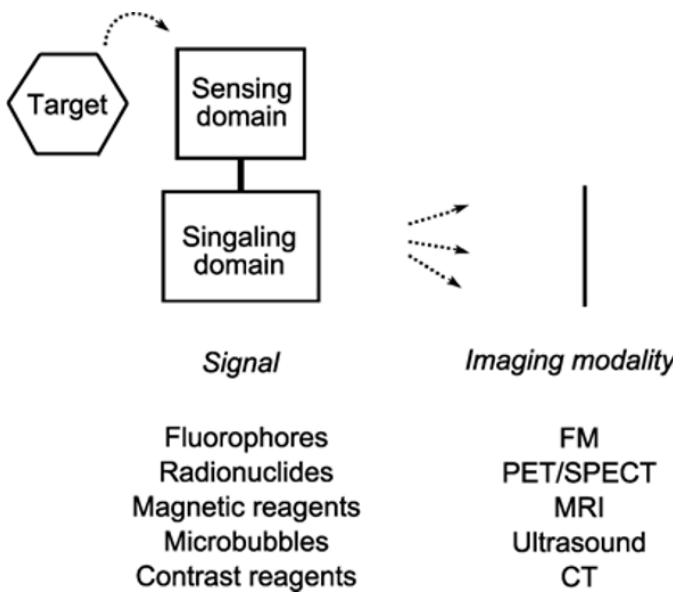

Figure 1. Biosensor for molecular imaging. A biosensor consists of a probe and an imaging modality. The probe has a sensing domain to bind a target molecule and a signaling domain to generate signals that can be imaged by an imaging modality. CT, Computed tomography; FM, fluorescence microscopy; MRI, magnetic resonance imaging; PET, positron emission tomography; SPECT, single photon emission computed tomography. ing high affinity (particularly slow off-rate), high specificity and high sensitivity to achieve high signal/noise ratio. It should also have good pharmacokinetic properties for clinical application which include high stability in vivo, low immunogenicity, low toxicity and easy cell and tissue penetration. Finally, a probe should be easily produced with low cost (Chen and Chen, 2010). Aptamers can meet all these criteria except for easy cell penetration due to their relatively large size (5-10 $\mathrm{kDa}$ ) in comparison to small molecules. Thus imaging cell surface molecules would be the most straightforward application for aptamer probes. However, for intracellular targets, an aptamer probe can be delivered into cells by transformation, transfection, microinjection or expression inside cells either independently or as an RNA tag.

Based on the types of target molecules imaged, different aptamer probes have been developed. For small molecule and protein imaging, an aptamer probe recognizes its target molecule by direct aptamer-target interaction (Fig. 2, 1-6). On the other hand, for RNA imaging, single or multiple copies of an aptamer probe need to be coupled to its target RNA either through physical linkage (Fig. 2, probe 7-9) or through hybridization (Fig. 2, probe 10-11). Individual examples of these probes are summarized in Table S1 and discussed in detail in the main text below.

Aptamer probes can be generated through rational design using aptamers that have been selected towards specific target molecules. In rational design, the most straightforward way is to link a reporter to an aptamer (Fig. 2, probe 1) or to the aptamer ligand (Fig. 2, probe 8a). Through specific binding, the reporter is enriched on the target molecule by way of the aptamer probe. The unbound reporters are then either washed away or confined in a particular compartment (e.g., nuclei) in the context of cell culture or cleared from body fluid and untargeted tissues in living organisms. The drawback of the probe 1 and $8 a$ is low signal/noise ratio due to insufficient separation of the bound from the unbound reporters. To increase signal/noise ratio, smart probes have been developed mostly based on fluorescence properties including fluorescence quenching (Fig. 2, probe 2-4), Förster resonance energy transfer (FRET) (Fig. 2, probe 5) or a "light-up" property (Fig. 2, probe 6, 7, 8b, 9 and 11). In fluorescence quenching, emission energy from a fluorophore donor is lost due to absorption by a proximate quencher. In FRET, emission energy from a donor fluorophore is used to excite a nearby acceptor fluorophore for fluorescence emission. Both fluorescence quenching and FRET are distance dependent. An aptamer probe can be designed such that the distance between fluorphore donor and fluorophore acceptor/quencher in the probe changes upon target binding. Fluorescence change due to distance change, either intramolecular (Fig. 2, probe 2, 3 and 5) or intermolecular (Fig. 2, probe 4) is then used to image a target molecule. For fluorescence light-up property, a fluorogen, which is nonfluorescent, can become fluorescent due to aptamer binding changing the microenvironment or the 
1<smiles>CCO</smiles>

2

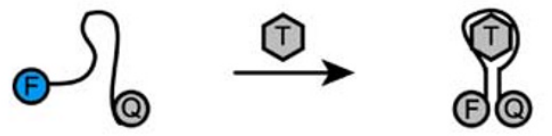

3

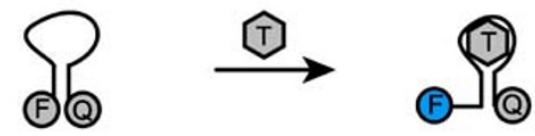

4

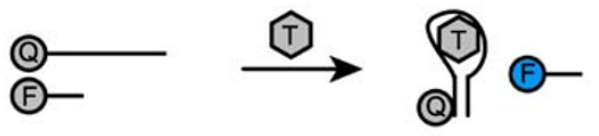

5

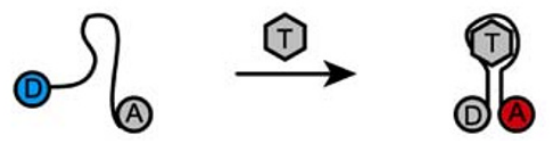

6

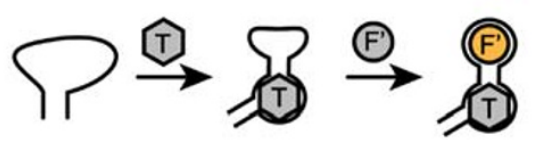

7

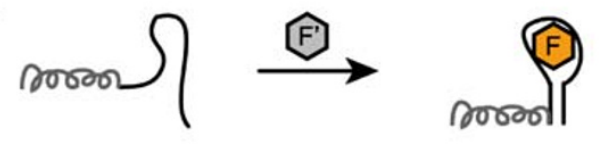

8

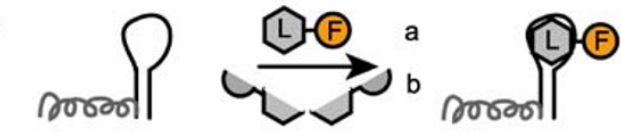

9

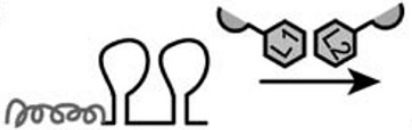

10
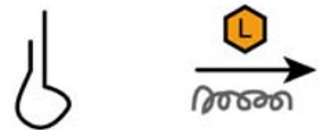

roser

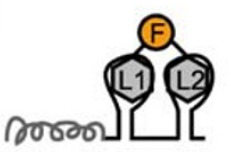

11

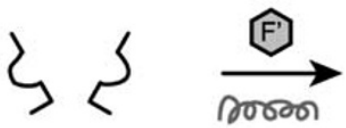

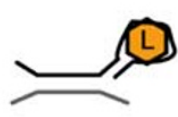

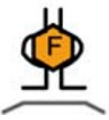

Figure 2. Probe design for aptamer-based molecular imaging. Aptamer probes have been developed to image a broad range of target molecules. For small molecule and protein imaging, an aptamer probe recognizes its target molecule (T) by direct aptamer-target interaction (probe 1-6). For RNA imaging, single or multiple copies of a probe need to be coupled to a target RNA (ग0000) either by physical linkage (probe 7-9) or by hybridization (probe 10-11). Signal from a probe can be either directly from a reporter conjugated to the probe (probe 1) or to the aptamer ligand (probes $8 a$ and 10) or through various mechanisms including Förster resonance energy transfer (probe 4), fluorescence quenching (probe 2), dequenching (probe 3 ) and light-up property (probe 7, 8b, 9, 11). Individual examples of these probes are summarized in Table S1 and discussed in detail in the main text. A, accepter; $\mathrm{D}$, donor; F, fluorophore; F', fluorogen; L, ligand; Q, quencher; $\mathrm{R}$, reporter; $\mathrm{T}$, target. chemical property of a fluorogen (Fig. 2, probe, 6, 7 and 11). Alternatively, split nonflurorescent proteins can become fluorescent due to reconstruction of functional fluorescent proteins upon aptamer binding (Fig. 2, probe $8 \mathrm{~b}$ and 9). In addition, aptamer modularity and allostery have been utilized in smart probe design too (Fig. 2, probe 6, 10 and 11).

In addition to the approach of rational design of existing aptamers, novel signaling aptamers can be generated by in vitro selection. One approach is to utilize the property that fluorescence is sensitive to the local chemical environment. In this approach, fluoresceinated uridine-incorporated RNAs were used to select ATP aptamers. The selected aptamers showed maximal twice increase in fluorescein fluorescence intensity due to the local chemical environment change upon ATP binding (Jhaveri et al., 2000). In another approach, the combination of structure switching and fluorescence quenching was used to in vitro select structure-switching signal aptamers. In the selection pool design, a constant region was flanked by two short random sequences with additional $5^{\prime}$ and $3^{\prime}$ constant regions for polymerase chain reaction (PCR) amplification. The generated aptamers were then captured by a resin-coupled ssDNA complementary to the central constant region of the aptamers. The aptamers were then eluted by target binding, which disrupted the hybridization between the aptamers and the resin-coupled ssDNA. After successful selection of the signaling aptamers, a final signaling probe consists of the selected aptamer, the short oligonucleotide that hybridizes to the middle of the aptamer and an additional short oligonucleotide that hybridizes to the terminal (either $5^{\prime}$ or $3^{\prime}$ end) of the aptamer. In the two short oligonucleotides, a fluorophore was linked to one and a quencher to the other. The fluorophore/quencher pair was positioned at proximity to have quenching effect. Binding of a target molecule to the probe displaces the middle hybridized short oligonucleotide and gives out a fluorescence signal (Nutiu and Li, 2005).

\section{Imaging modality}

Depending on the types of reporters/contrast reagents, different imaging modalities have been used in molecular imaging. These imaging modalities have been comprehensively reviewed (Massoud and Gambhir, 2003; Weissleder and Pittet, 2008; Pysz et al., 2010). Here briefly described is the principles of these imaging modalities in the context of aptamer-based molecular imaging. Most of the imaging modalities utilize the probe 1 design in Fig. 2 to achieve targeted imaging except fluorescence microscopy, which can take advantage of all the probe design in Fig. 2 for best performance (Table S1).

Among the imaging modalities, fluorescence microscopy is the most widely used in basic research mostly in a cellular context. Fluorescence microscopy in a cellular context has temporal resolution of picoseconds to seconds (Suhling et al., 
2005), spatial resolution of $10-200 \mathrm{~nm}$ and sensitivity of up to a single molecule (Toomre and Bewersdorf, 2010). However, whole-body fluorescence microscopy on animals only has temporal resolution of seconds to minutes, spatial resolution of millimeters and depth penetration of 1-6 $\mathrm{cm}$ due to light absorption by tissues. The reporters in fluorescence microscopy are fluorophores with emission of either visible light or near-infrared light, which can be detected by a photomultiplier detector. Fluorescence microscopy is the dominant imaging modality for aptamer-based molecular imaging mostly in a cellular context and with some success on animal models (Table S1).

Imaging modalities from nuclear medicine have been applied in diagnostic molecular imaging, mostly on living organisms. For radionuclide reporters, 2D scintigraphy or 3D tomography can be used for imaging. The most commonly used 3D tomographies for radionuclide imaging are positron emission tomography (PET) and single photon emission computed tomography (SPECT). In PET, radionuclides including ${ }^{18} \mathrm{~F},{ }^{68} \mathrm{Ga},{ }^{89} \mathrm{Zr}$ and ${ }^{124} \mathrm{I}$ emit positrons to collide with electrons in a living organism to produce pairs of high-energy $\mathrm{y}$-ray. In SPECT, radionuclides including ${ }^{67} \mathrm{Ga},{ }^{99 \mathrm{~m}} \mathrm{Tc},{ }^{111} \mathrm{In}$ and ${ }^{123}$ I emit low-energy $y$-ray. With the combination of computed tomography with imaging $Y$-ray by a $Y$-camera, 3D imaging can be achieved for both PET and SPECT without depth limitation and with temporal resolution of seconds to minutes and spatial resolutions of 1-2 $\mathrm{mm}$. PET has a detection limit of $10^{-12}-10^{-11} \mathrm{~mol} / \mathrm{L}$, which is an order of magnitude more sensitive than SPECT. In aptamer-based molecular imaging, radionuclides including ${ }^{99 \mathrm{~m}} \mathrm{Tc}$ and ${ }^{67} \mathrm{Ga}$ have been used as reporters for 2D-scintigraphy (Charlton et al., 1997; Hicke et al., 2006; Hwang do et al., 2010).

In computed tomography (CT), 3D images are reconstructed from a series of $2 \mathrm{D}$ X-ray images taken rotating around a single axis. There is no depth limit for CT with temporal resolution of minutes and spatial resolution of 50-200 $\mu \mathrm{m}$. The sensitivity of CT is not well defined. Based on the ability of gold to induce $\mathrm{X}$-ray attenuation, a prostate specific membrane antigen (PSMA) aptamer-conjugated gold nanoparticle was used as a contrast reagent to image PSMA-positive LNCaP cells in vitro (Kim et al., 2010).

Ultrasound are sound waves with frequencies of $>20 \mathrm{kHz}$. The 3D images can be constructed from ultrasound imaging based on the reflection signature of the subjects. Ultrasound imaging has depth limitation of millimeter to centimeters with temporal resolution of seconds to minutes and spatial resolution of $50-500 \mu \mathrm{m}$. Based on the property that air-bubbles can scatter ultrasonic energy, an aptamer-conjugated nanobubble was used as a contrast reagent to image CCRF-CEM cells in cell culture (Wang et al., 2011).

Magnetic resonance imaging (MRI), another in vivo imaging modality, is based on nuclear magnetic resonance. MRI has no depth limit with temporal resolution of minutes to hours and spatial resolution of 25-100 $\mu \mathrm{m}$. The sensitivity of
MRI is low $\left(10^{-5}-10^{-3} \mathrm{~mol} / \mathrm{L}\right)$. In MRI, a strong magnetic field is applied to align magnetized nuclei. The aligned magnetic nuclei can be altered by a resonance frequency from a gradient radio frequency transmitter when it is on. When the radio frequency transmitter is off, the relaxations of the nuclei spin are recorded to produce 3D images. The relaxations of nuclei in different tissues are different, thus producing contrast imaging, which can be further enhanced by contrast reagents. Aptamer conjugated contrast reagents can achieve targeted MRI. Aptamer conjugated magnetic beads, $\mathrm{CoFe}_{2} \mathrm{O}_{4}$, $\mathrm{Fe}_{3} \mathrm{O}_{4}$ nanoparticles have been applied in targeted imaging in animal models (Hwang do et al., 2010; Yu et al., 2011).

\section{Signal amplification}

To achieve high signal/noise ratio, sensitivity and resolution in imaging, different approaches have been used.

The signal/noise ratio can be increased either by decreasing noise or increasing the signal or both. To this end, aptamer probes usually utilize fluorescence properties including fluorescence quenching (Fig. 2, probe 2-4), FRET (Fig. 2, probe 5) and light-up fluorophores (Fig. 2, probe 6, 7, $8 \mathrm{~b}, 9$ and11). In addition, the binding of an aptamer probe to its target can enrich reporters at the site, thus increasing the local signal strength (Fig. 2, probe 1, 8a and 10).

Another way to amplify signal is through multivalency. This is achieved through either multivalency of aptamer or multivalency of the reporters. For example, single molecule imaging of mRNA has been achieved by tagging an mRNA with 24 units of MS2 aptamers, which accumulate MS2-GFP fusion proteins on the mRNA (Fusco et al., 2003). On the other hand, a nanoparticle doped with thousands of fluorescent dyes can greatly increase the signal by several orders of magnitude (Knopp et al., 2009). Such nanoparticle-aptamer conjugates, if biocompatible, have the potential to dramatically amplify the signal for sensitive imaging.

Although enzymatic amplification (substrate conversion, PCR, rolling circle amplification, aptazyme) has been achieved in vitro for biosensors (Zhou et al., 2010), little has been done with these in aptamer-based molecular imaging. Allosteric aptazymes may have the potential for use in signal amplification for aptamer-based molecular imaging. This requires further investigation.

\section{Multiplex imaging}

Multiplex imaging has two aspects. One is to image multiple molecules simultaneously and the other is to image a single molecule with multiple imaging modalities. The former is important for understanding molecular interactions and the interplay of multiple events in biological systems and for diagnostic imaging of disease signatures. The latter offers the opportunity to study the different aspects of a single molecule event by different imaging modalities and the flexibility of a 
broad range of imaging and the potential to overcome the shortcomings and utilize the strength of individual imaging modalities.

Aptamer-based multiplex imaging of multiple molecules simultaneously has been achieved. To study the trafficking of two mRNAs ASH1 and IST2 in yeast cell, 12× Box and 6x MS2 aptamers were used as tags for the mRNAs respectively. The expressed tagged mRNAs were then imaged by AN22-GFP and MS2-RedStar respectively and their trafficking was monitored in real-time. As two localized mRNAs, ASH1 and IST2 were cotransported directionally by a common ribonucleoprotein particle to the target site but not the nonlocalized mRNA HOM2 (Lange et al., 2008). In addition to trafficking, spatial distribution of multiple molecules can be studied simultaneously by aptamer imaging as well. Nucleolin and $\alpha_{4} \beta_{3}$ integrin, two proteins highly expressed in many cancer cells, were imaged simultaneously on their cellular distribution by a quantum dot 655 (QD655) conjugated with a nucleolin-binding aptamer AS1411 and a QD605 conjugated with an $\alpha_{v} \beta_{3}$ integrin-binding arginine-glycine-aspartic acid (RGD) peptide (Ko et al., 2009). On the other hand, an RGD-conjugated QD525 (the fluorophore donor) and an AS1411-conjugated Cy3 (the fluorophore acceptor) were used together to image the colocalization of nucleolin and $\alpha_{v} \beta_{3}$ integrin based on FRET. Although nucleolin and $\alpha_{v} \beta_{3}$ integrin were highly coexpressed, they were only partially colocalized in HeLa cells (Kang et al., 2009b). To study cancer marker signature, three different aptamers (TTA1 aptamer against tenascin-C, AS1411 aptamer against nucleolin and MUC-1 aptamer against MUC-1) were conjugated respectively to QDs with different emission wavelengths (605 $\mathrm{nm}, 655 \mathrm{~nm}, 705 \mathrm{~nm}$ ). The aptamer-QD conjugates were then applied simultaneously to image the expression patterns of the three proteins in different cancer cell lines by confocal microscopy. Different cancer cell lines had different expression signatures, among which $\mathrm{C} 6$ and HeLa cell lines showed coexpression of the three proteins on the cell surface of a single cell. This indicates that multiplex aptamer imaging has the potential for obtaining protein expression signatures for cancer diagnosis (Kang et al., 2009a).

Another aspect of aptamer-based multiple molecule imaging is to image protein-protein/RNA interaction in real time. To study the interaction of fragile $X$ mental retardation protein (FMRP) to its native RNA binding site, an MS2 aptamer tag was inserted near the FMRP native binding site in an mRNA. With coexpression of MS2-split Venus and FMRP-split Venus, fully functional Venus was reconstructed and gave out fluorescence when FMRP-split Venus binding to its native RNA site brought together the MS2-split Venus binding to the MS2 aptamer tag nearby. To determine the interaction of two RNA binding proteins, FMRP and human zipcode-binding protein 1 ortholog IMP1, a vector was constructed to contain a MS2 aptamer tag without RNA binding sites for FMRP and IMP. The MS2-FMRP-split Venus could recruit IMP1-split Venus but not control protein to give out fluorescence and vice versa. This indicated that FMRP-IMP1 interaction was independent of their RNA binding sites and the interaction of the two proteins could be imaged by Venus fluorescence (Rackham and Brown, 2004).

In addition to multiplexing on molecules, multiplexing on imaging modalities has been done. To achieve multiple modality imaging of a single probe, a nanoparticle consisting of cobalt ferrite, fluorescent rhodamine and ${ }^{67} \mathrm{Ga}$-citrate was conjugated to an aptamer AS1411 for targeting nucleolin. The developed probe was applied in cell culture to image nucleolin-expressed C6 glioma cells by fluorescence microscopy. In vivo imaging of the tumor model in nude mice was also achieved by scintigraphy with ${ }^{67} \mathrm{Ga}$ as a reporter and by MRI imaging with cobalt ferrite as a reporter (Hwang do et al., 2010).

Finally, it is possible to integrate aptamer imaging with therapy. A probe was developed to contain a quantum dot, a PSMA aptamer and a doxorubicin. The probe was internalized into the prostate cancer cell line LNCaP after it bound to cellular surface PSMA. After probe internalization, doxorubicin was released from the probe to kill the cancer cells and both quantum dot and doxorubicin became unquenched and fluorescent for cell imaging (Bagalkot et al., 2007). In another study, a gold nanoparticle was conjugated to a PMSA aptamer loaded with doxorubicin. The gold nanoparticle, as a targeted contrast reagent for $\mathrm{CT}$ imaging, showed four times higher intensity in PMSA-expressing cell (LNCaP) than that in PMSA-negative cell (PC3 cells). The probe also selectively inhibited growth of PMSA-positive cells in cell culture (Kim et al., 2010). Yet another probe was developed with a superparamagnetic iron oxide nanoparticle conjugated with a PMSA aptamer and loaded with doxorubicin. The probe was used to image LNCaP cells both in vitro by fluorescence microscopy and in vivo on a tumor-bearing mice model by MRI. The probe accumulated on the tumor site and effectively inhibited tumor growth in vivo. Thus the probe had the capability of imaging the tumor, targeted delivery of drug and simultaneously monitoring the therapeutic effect ( $\mathrm{Yu}$ et al., 2011).

To conclude the first part of the review, aptamer-based biosensor development has been discussed on probe design, imaging modality and multiplex imaging in the context of molecular imaging. In the second part of the review, application of aptamer-based biosensors for imaging different types of target molecules will be highlighted.

\section{TARGETS DETECTED BY APTAMER-BASED MOLECULAR IMAGING}

Aptamer-based molecular imaging has been applied to image a broad range of target molecules including small molecules, RNAs and proteins. Most of the imaging studies are done in the context of cell culture and some in animal models (Table S1). 


\section{Small molecules}

Small molecules including ions and metabolites play important roles in cell metabolism and function. However, there are limited tools available for sensing, imaging and quantifying small molecules in vivo. Some ions and metabolites can be imaged specifically inside cells with genetically encoded fluorescent protein sensors but such sensors are difficult to be engineered for a broad range of small molecules (Bermejo et al., 2011; Palmer et al., 2011). On the other hand, aptamers can be selected for a broad range of target molecules and each aptamer is specific to its target molecule. Thus there is great potential for wide application of aptamer-based small molecule imaging in studying cellular metabolism. There have already been some attempts of imaging $\mathrm{K}^{+}$, ADP, ATP and S-adenosylmethionine (SAM) with aptasensors.

To image $\mathrm{K}^{+}$inside cell, a probe (Fig. 2, probe 5) using the thrombin aptamer (with G-quartet structure) as the recognition element was developed based on the fact that G-quartet structure is sensitive to $\mathrm{K}^{+}$. In the probe (PSO-5), a biotinylated neutral peptide was linked to the $5^{\prime}$ end of the thrombin aptamer with TAMRA fluorophore linked to the probe at the peptide site and FAM fluorophore at the $3^{\prime}$ end of the thrombin aptamer. Then a biotinlyated nuclear export signal peptide (B-NES) was synthesized. Both PSO-5 and B-NES could be captured by streptavidin, a protein with four biotin binding sites. With a ratio of 1:3:1 of PSO-5, B-NES and streptavidin, a ternary complex formed. This ternary complex was retained in the cytoplasm instead of the nucleus for $\mathrm{K}^{+}$ imaging. In the absence of $\mathrm{K}^{+}$, the thrombin aptamer was unstructured and TAMRA and FAM were far away from each other. In the presence of $\mathrm{K}^{+}$, the thrombin aptamer formed a G-quartet structure, bringing TAMRA and FAM close enough to generate FRET signal for $\mathrm{K}^{+}$imaging. When introduced into HeLa Cells, the ternary complex imaged cytosolic $\mathrm{K}^{+}$ concentration and dynamics spatiotemporally in real-time. The probe also had $>200$ times selectivity of $\mathrm{K}^{+} / \mathrm{Na}^{+}$(Ohtsuka et al., 2012).

ATP, the cellular energy source and a substrate for various signal transduction and metabolism, is another molecule actively studied. An aptamer nano-flare (Fig. 2, probe 4) was developed to image and quantify cellular ATP concentration. In the aptamer nanoflares, ATP aptamers were conjugated to a gold nanoparticle and hybridized to Cy5-labeled complementary DNA reporters. The Cy5 fluorescence is quenched by the gold nanoparticle. When ATP binds to its aptamer, the hybridized Cy5-DNA reporters are released and the quenched Cy 5 becomes fluorescent. The aptamer nanoflares could readily enter HeLa cells and selectively measure intracellular ATP concentration at $0.1-3 \mathrm{mmol} / \mathrm{L}$ range (Zheng et al., 2009). Instead of direct conjugation, an ATP aptamer labeled with carboxyfluorescein (FAM) was absorbed by a grapheme oxide nanosheet to form an aptamer/grapheme oxide nanocomplex, in which FAM fluorescence was quenched by the nanosheet. Upon ATP binding, the FAM labeled ATP aptamer could be released from the nanocomplex and became fluorescent. The aptamer/grapheme oxide complex was applied to image intracellular ATP in cell culture (Wang et al., 2010). In another study of imaging and quantifying ATP in yeast cells, a probe (Fig. 2, probe 3 ) based on ATP aptamer was embedded inside a polyacrylamide nanoparticle. Texas red (the reporter) and Black Hole 2 (the quencher) were conjugated to the $5^{\prime}$ and $3^{\prime}$ ends of the probe and were close to each other due to a hairpin structure. Binding of ATP to the ATP aptamer in the probe changed the hairpin structure, resulting in Texas red moving away from Black Hole 2 and therefore increasing fluorescence. The probe was applied to image and measure total intracellular ATP concentration (2.9-5.1 mmol/L) (Nielsen et al., 2010). Using the same design but with a newly selected ATP aptamer that can distinguish ATP from ADP and AMP and the reporter-quencher pairs of Alexa Fluor 488-Black Hole 1 and Texas red-Black Hole 2, the same group developed new probes to image and measure the dynamics of intracellular ATP in yeast with time resolution of seconds and ATP concentrations of $0.5-8 \mathrm{mmol} / \mathrm{L}$. The ATP concentration oscillated and varied under different conditions (Ozalp et al., 2010a, 2010b).

Finally, based on the concept of aptamer modularity and allostery (Stojanovic and Kolpashchikov, 2004), aptasensors have been developed to image the dynamics of ADP and SAM in real time in E. coli (Paige et al., 2012) (Fig. 2, probe 6; Fig. 3A). In the aptasensors, there is a sensing aptamer domain, a transducer (a weak stem structure) and an activatable signaling aptamer domain. The sensing aptamer domain can recognize a small molecule target (e.g., ADP or SAM). Without binding of the small molecule target to the sensing aptamer domain, the signaling aptamer domain (e.g., modified spinach aptamer) is unstructured and cannot bind to its ligand [e.g., 3,5-difluoro-4-hydroxybenzylidene imidazolinone (DFHBI)], the reporter. When the small molecule target binds the sensing aptamer domain, the transducer is stabilized, which facilitates the signaling aptamer domain to form its stable conformation for binding to the reporter for generating signal for imaging the small molecule target. Here the light-up property of spinach-DFBHI interaction is utilized for imaging, in which DFBHI is a fluorogen and fluoresces upon spinach binding (Paige et al., 2011). This allosteric aptasensor can potentially be a general approach for small molecule imaging by incorporating different sensing aptamer domains for different small molecule targets.

\section{RNAs}

RNAs are essential components in cells. They include messenger RNA (mRNA), ribosomal RNA (rRNA), transfer RNA (tRNA), small nuclear RNA (snRNA), microRNA (miRNA), small interference RNA (siRNA), antisense RNA and other 

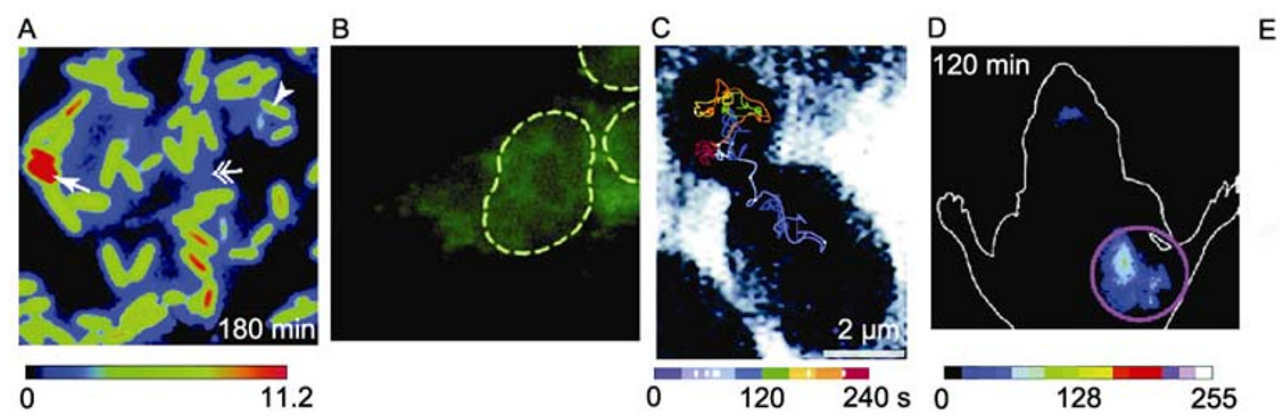

E

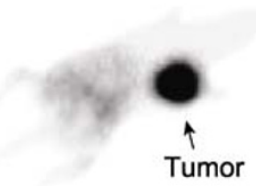

Figure 3. Some examples of aptamer-based molecular imaging. (A) Imaging cellular S-adenosylmethionine (SAM) in live $E$. coli by fluorescence microscopy using the probe 6 in Fig. 2. Only the image taken at 180 min after the treatment of methionine, the SAM precursor is shown here. The image is color-coded to show the fold increase in fluorescence intensity relative to 0 min. SAM level is heterogeneous among cells with some higher than average (arrow), some slow in increase (arrow head) and some first increase and then decrease (double arrow). (from part of the Fig. 1c in Paige et al., 2012). (B) Imaging 5S RNA tagged with spinach in live HEK293 T cells by fluorescence microscopy using the probe 7 in Fig. 2. The nuclear borders are shown with dash-lines. (from part of the Fig. 4c in Paige et al., 2011). (C) Imaging Ash1 mRNA movement using MS2-GFP system by fluorescence microscopy using the probe $8 \mathrm{a}$ in Fig. 2. The diagram shows the moving trajectory of an Ash1 mRNP particle from a mother cell to a bud in 240 s over $43 \mu \mathrm{m}$. (from Fig. 4G in Bertrand et al., 1998). (D) Imaging protein tyrosine kinase-7 (PTK7), a marker of CCRF-CEM tumor, in a CCRF-CEM tumor-bearing mouse by a fluorescence imaging system using the probe 3 in Fig. 2 . Only the image taken 120 min after the injection of the aptamer probe is shown here. The fluorescence intensity is color-coded and the tumor is highlighted with a pink circle. (from part of the Fig. 5A in Shi et al., 2011). (E) Imaging tenascin-C, a tumor marker, in a MDA-MB-435 breast tumor bearing nude mice by 2D-sctintigraphy using the probe 1 in Fig. 2. (from part of the Fig. 6 in Hicke et al., 2006). The figures above are reprinted with permission from the American Association for the Advancement of Science (A and B), Elsevier (C), the National Academy of Sciences of the United States of America (D) and the Society of Nuclear Medicine and Molecular Imaging (E).

noncoding RNA (ncRNA). They are involved in all aspects of cellular activities including transcription, RNA splicing, RNA modification, translation and gene regulation. To understand RNA functions, it is important to elucidate its metabolism, dynamics, trafficking and cellular localization. One way to tackle these issues is by molecular imaging of RNA molecules. RNA imaging has been achieved at the single molecule level using hybridization-based probes including fluorescence in situ hybridization (FISH) and molecular beacons (Bao et al., 2009; Itzkovitz and van Oudenaarden, 2011). These hybridization probes can detect endogenous RNAs without tagging but usually require invasive delivery of the probes. Instead of FISH and molecular beacons, the main focus here is on aptamer-based RNA imaging, a different imaging methodology. In aptamer-based RNA imaging, one or multiple copies of an RNA aptamer tag are usually inserted into the untranslated region of a target RNA. The target RNA is then imaged by the aptamer tag-ligand binding. The ligand can be an activatable fluorogen ligand, a fluorophor-conjugated ligand, a split fluorophore-conjugated split ligand or a split fluorophore conjugated with two ligands (Fig. 2, probe 7-9). Imaging of endogenous RNAs by aptamer probes based on hybridization has also been proposed and demonstrated as proof-of principle in test tube, but it has not been implemented in a cellular context or in vivo (Fig. 2, probe 10-11). Different types of aptamer-based RNA imaging are discussed below with specific examples referenced.

\section{RNA imaging based on aptamer tag bound by ligand-fused fluorescent proteins}

Different aptamer-fluorescent protein pairs have been developed for real time RNA imaging in cell culture. In this approach, an aptamer tag is inserted into a target RNA and the aptamer ligand is fused to a fluorescent protein. The target RNA is then imaged by the fluorescent protein through aptamer-ligand interaction. The aptamer-fluorescent protein pairs include MS2-GFP/paGFP/YFP/RedStar/mRFP/split Venus, $\lambda$ N22-GFP/RedStar, U1A-GFP, split elF4A- split GFP and HTLV-1 Rex/ $\lambda$ N22-split EGFP. A comparison of these pairs is listed in Table 1. Recently, aptamers have been developed to directly bind different fluorescent proteins (Shui et al., 2012). Such pairs may have the potential in imaging without fusing a ligand to a fluorescent protein.

In the imaging system, multiple copies of an aptamer tag are inserted into the untranslated regions of a target RNA with coexpression of a fluorescent protein fused with a ligand that can bind to the aptamer tag on the target RNA. The insertion site of the aptamer tag into the target RNA should be carefully selected and tested with minimal interference of the function, stability and trafficking of the target RNA. To achieve good signal/noise ratio, the optimal expression level of the target RNA and the ligand-fused fluorescent protein needs to be empirically determined. To decrease background, a nuclear localization signal is usually attached to the fluorescent 
Table 1 The properties of aptamer-fluorescence protein (FP) pairs in RNA imaging

\begin{tabular}{|c|c|c|c|c|}
\hline Ligand & Aptamer tag & $\mathrm{K}_{\mathrm{d}}(\mathrm{nmol} / \mathrm{L})$ & Fusion FP & Applicability \\
\hline MS2 (129 aa) & $\begin{array}{l}\text { ACAUGAGGAU(U/C)ACCCAUGU } \\
19 \text { nt/aptamer, 4-24 aptamers/tag }\end{array}$ & $<5$ & $\begin{array}{l}\text { MS2-FP, } \\
\text { MS2-split FP }\end{array}$ & Broad cell types \\
\hline AN22 (22 aa) & $\begin{array}{l}\text { GCCCUGAAAAAGGGC } \\
15 \text { nt/aptamer, 4-12 aptamers/tag }\end{array}$ & 22 & $4 \times \lambda N 22-3 x E G F P$ & Broad cell types \\
\hline $\begin{array}{l}\text { HTLV-1 Rex (16 aa) } \\
\quad \& \\
\text { AN22 (22 aa) }\end{array}$ & $\begin{array}{l}\text { AGGCGACGGTACGCAAGTACTCTTGCGCCGGCCT, } \\
34 \text { nt/aptamer, } 1 \text { aptamer/tag } \\
\text { GGCCUAGGGCCCUGAAAAAGGGCCCUUUCC, } \\
30 \text { nt/aptamer, } 1 \text { aptamer/tag }\end{array}$ & $\begin{array}{l}30 \\
22\end{array}$ & $\begin{array}{l}\text { Rex-NEGFP } \\
\quad \& \\
\lambda \text { N22-CEGFP }\end{array}$ & Broad cell types \\
\hline U1A (95-102 aa) & $\begin{array}{l}\text { GAAGAGCCAUUGCACUCCGGUUCUUC } \\
18 \mathrm{nt} / \text { aptamer, } 4-16 \text { aptamers/tag }\end{array}$ & $0.1-4$ & U1A-GFP & Bacteria and yeast \\
\hline elF4A (406 AA) & $\begin{array}{l}\text { GGGGACCGCGCCCCACAUGUGAGUGAGGCCGAAAC } \\
\text { GUAGAUUCGACAGGAGGCUCACA, } 58 \text { nt/aptamer, } 2 \\
\text { aptamer/tag }\end{array}$ & 27 & $\begin{array}{l}\text { elF4A(1-215)-NEGFP } \\
\text { and elF4A(216-406)- } \\
\text { CEGFP }\end{array}$ & Bacteria \\
\hline
\end{tabular}

Note: source of aptamer sequence and affinity. MS2 aptamer (Lim and Peabody, 1994), AN22 aptamer (Austin et al., 2002), HTLV-1 Rex aptamer (Yiu et al., 2011), U1A aptamer (Zeng and Hall, 1997) and elF4A aptamer (Oguro et al., 2003).

protein to localize the protein in the nucleus, thus not interfering with imaging in the cytoplasm. Another way to decrease background is to use split fluorescent proteins, which will fluoresce only when reconstructed by aptamer binding. The drawback of split fluorescent proteins is that the interaction of the two protein halves is usually irreversible and not suited for imaging RNA dynamics.

Aptamer-fluorescent protein imaging systems have been used to study transcription, RNA processing, transport, localization, translation and turnover at the single molecule and single cell level. Unlike the average population behavior, single molecule and single cell shows heterogeneity and diversity of individual molecules and cells. This will be discussed in more detail below.

To study mRNA transcription dynamics, a developmental gene dscA was tagged with 24 units of MS2 aptamer for RNA imaging in social amoeba Dictyostelium. The transcription of dscA gene was discontinuous with a pulsing mode of on and off (mean interval and duration of 5-6 min) and various percentages $(5 \%-40 \%)$ of cells expressing dscA. Transcription memory and synchronism in transcription initiation in adjacent cells was also observed for dscA gene (Chubb et al., 2006). To further determine the transcription pulse behaviors among genes, transcription of five housekeeping genes and five developmental genes were studied in Dictyostelium using MS2-GFP tagging system. Distinct transcription pulse duration (mostly $<10 \mathrm{~min}$ ), frequency (2-6 pulses/h) and intensity (0-8 folds difference) among the genes with various percentage of cells $(1 \%-90 \%)$ expressing the genes. With the progress of development stages, the percent of cells expressing the genes increased for most developmental genes but decreased for most of the housekeeping genes. For transcription pulse behavior (pulse duration, frequency and intensity) at different development stages, most development genes had constant transcription pulse behavior while housekeeping genes showed tunable transcription pulse behavior (Muramoto et al., 2012). To measure the mRNA transcription kinetics (kinetic parameters in promoter binding, initiation and elongation), a gene array of 200 copies of a gene cassette (CFP-SKL) each tagged with 24 units of MS2 aptamer was inserted into a single locus on a chromosome of human U2OS cells. The transcription of the gene cassette was imaged with coexpressed YFP-Pol II (for Pol II imaging) and MS2-GFP (for nascent mRNA imaging). The onset of transcription was very inefficient. In the transcription onset, $13 \%$ of Pol II binding to promoters proceeded to initiation step, among which only $8.6 \%$ engaged in elongation, resulting a net $1 \%$ of $\mathrm{Pol}$ II in successful transcription. The mean residence time of Pol II for promoter binding, initiation and elongation were 6, 54 and $\sim 500 \mathrm{~s}$ respectively for this $3.3 \mathrm{~kb}$ gene. In the elongation stage, there was a rapid elongation at the rate of $72 \mathrm{nt} / \mathrm{s}$ with a probabilistic (4.2\%) long pausing (4-5 min) for individual elongating Pol II. This resulted in a probability of $26 \%$ Pol II pausing at a single gene locus. The elongation pausing kinetics of Pol II (Darzacq et al., 2007) and the potential different initiation rate of Pol II (Muramoto et al., 2012) may explain the mRNA transcriptional pulsing dynamics. To correlate chromatin structure with transcription, a 200-copy gene array system as mentioned above with additional tetracycline inducible elements was integrated into chromosome 1 heterochromatic region in human U2OS cells. Each copy of the gene array had 256 copies of Lac operator and 24 copies of MS2 aptamer coding sequences. Chromatin structure was thus imaged with CFP-Lac repressor, mRNA with MS2CP-YFP and proteins (Histones and HP1) with tagged YFP. The histone exchange (HP1a depletion and H3.3 deposition) transformed the gene array from a silenced mode into an active mode of transcription. The transcription showed a dynamic mode of rapid increase upon induction, peak and then decrease over time. This imaging system allowed to integrate the real-time imaging of chromatin structure with transcription activation (Janicki et al., 2004). Transcription dynamics and diversity in individual E. coli. cells have also been studied by both MS2-GFP and split elF4A-GFP system. It was proposed that the dynamics of 
promoter open complex formation resulted in transcription dynamics in individual cells, which led to translation diversity based on MS2-GFP imaging (Smolander et al., 2011). In split elF4A-GFP study, it was shown that upon induction, $50 \%$ of cells showed a rapid increase in transcription which peaked at 50-70 $\mathrm{min}$ and then decreased to basal level at 75-125 min while another $50 \%$ of cells showed no response to induction (Valencia-Burton et al., 2009).

Eukaryotic mRNAs need cotranscriptional/posttranscriptional processing including RNA splicing in the nucleus before their export to the cytoplasm. In mRNA splicing, introns are removed and exons are joined to form mature mRNAs. To image cotranscriptional splicing in vivo in human U2OS cells, a model system with 4 units of MS2 aptamer in the intron (MINX construct) was constructed. The kinetics of splicing was measured by fluorescence recovery after photobleaching with mean splicing time of $162 \mathrm{~s}$. The splicing kinetics was best fit with a three-step model in which splicing was regulated by three successive rate-limiting steps (Schmidt et al., 2011). In addition, U1A-GFP system has been used to study the relationship of pre-mRNA processing and nuclear export in yeast. Different mRNAs (Rpl25, Pgk1 and Ssa4) had different maturation pathways but utilized the same export machinery. Mutations in export machinery and $3^{\prime}$ processing (polyadenylation) abolished nuclear export of all the mRNAs tested. However, defects in splicing machinery only affected the export of mRNA with introns (Rpl25). In addition, different ribonucleoprotein (RNP) packages affected RNA export, for example, Hrp1 as a component of RNP of a heat-shock RNA (Ssa4) facilitated only heat-shock RNA export but not non-heat-shock RNA (Rpl25 and Pgk1) export under ethanol stress (Brodsky and Silver, 2000).

After processing, mature mRNAs are exported from the nucleus to the cytoplasm and their corresponding locations. With CFP mRNA as a model, the mRNA movement in the nucleus was studied by MS2-YFP system. CFP mRNP movement followed simple diffusion model with a velocity of $\sim 0.55 \mu \mathrm{m} / \mathrm{s}$ and a diffusion coefficient of $0.04-0.09 \mu \mathrm{m}^{2} / \mathrm{s}$. Among all the CFP mRNPs, $58 \%$ of the mRNPs showed diffusive movement, $42 \%$ corralled and $<1 \%$ static. The corralled movement might reflect the heterogeneous nature of nucleoplasm (Shav-Tal et al., 2004). In the cytoplasm, single mRNA movement was proposed to follow a stochastic model based on imaging studies by MS2-EGFP system. In the model, mRNAs move along the cytoskeleton with continuously switching among static (anchoring), diffusional and directed (active transport) modes with sequence-dependent probabilities. Corralled movement of mRNA probably results from the restricted diffusion of mRNA in cytoplasmic microdomains. Localization signal on mRNA (e.g., zipcode for $\beta$ actin) can increase the frequency and length of directional movement without changing the velocity, thus facilitating mRNA transport and localization (Fusco et al., 2003). This model has been supported by mRNA transport and localiza- tion studies on ASH1 (Fig. 3C) and IST2 in yeast (Bertrand et al., 1998; Lange et al., 2008), Bcd (Weil et al., 2006) and Oskar (Zimyanin et al., 2008) in Drosophila oocytes, $\beta$-actin in chicken embryonic fibroblast cells (Yamagishi et al., 2009) and Arc (Dynes and Steward, 2007) and CaMKlla (Rook et al., 2000) in rat/mouse neurons using MS2/AN22-fluorescent protein imaging systems. In the studies, the untranslated regions ( $5^{\prime}$ and $3^{\prime}$ UTR) determined the mRNA transport efficiency and localization. The mRNAs moved discontinuously and stochastically with velocities ranging from $20 \mathrm{~nm} / \mathrm{s}$ to $1 \mu \mathrm{m} / \mathrm{s}$ depending on its modes of diffusion or active transport on different molecular motors (actin or microtubule). In addition to nuclear export, mRNAs (PGK1 and MFA2P) moved between different organelles in cytoplasm, for example, between polysomes and P-bodies as shown by U1A-GFP tagging system (Brengues et al., 2005). mRNA transport was also coupled to translation repression during transport (Rackham and Brown, 2004) and regulated local translation (Bi et al., 2006; Daigle and Ellenberg, 2007; Dictenberg et al., 2008). Finally, the aptamer-fluorescent protein imaging systems (MS2-GFP and split elF4A-EGFP) was applied to show the differential spatiotemporal localization of $E$. coli RNAs (Valencia-Burton et al., 2007; Valencia-Burton et al., 2009; Nevo-Dinur et al., 2011), the pri-miRNA localization in D-body in Arabidopsis (Fang and Spector, 2007) and the binding dynamics of noncoding Xist RNA to X-chromosome ( $\mathrm{Ng}$ et al., 2011).

RNA turnover is another aspect of RNA metabolism. MS2-GFP system was applied to show the direct relationship between nuclear retention and degradation of LYS2 mRNA in the nucleus in yeast (Das et al., 2006). With the caveat that the aptamer tag has no effect on RNA turnover (stability and degradation), the aptamer-fluorescent protein imaging systems have the potential application in studying RNA turnover in real-time in vivo.

\section{RNA imaging based on aptamer-fluorogenic dye pair (light-up probes)}

In aptamer-fluorescent protein system for RNA imaging, the large size of the fluorescent protein ( $27 \mathrm{kDa}$ for each GFP) may interfere with RNA properties including processing, trafficking and turnover. Moreover the fluorescence from unbound fluorescent proteins can cause significant high background in imaging. In addition, real-time and dynamic RNA imaging can be affected by the long maturation time during synthesis and the slow turnover rate of the fluorescent proteins. To overcome these shortcomings, small molecules, particular fluorogenic dyes, have been proposed to replace fluorescent proteins for real-time and dynamic RNA imaging. For small molecules to meet with imaging requirement, they need to be permeable to cell membrane, nontoxic, bind avidly and specifically to their aptamers but have low nonspecific binding to cellular components and low in background signal. Efforts in this direction are listed in Table 2 and will be described below. 
Table 2 The properties of aptamer-fluorogen pairs for RNA imaging

\begin{tabular}{lllll}
\hline Fluorogen & Aptamer length & $\mathrm{K}_{\mathrm{d}}$ & Fluorescence increase by times & Reference \\
\hline Malachite green & $38 \mathrm{nt}$ & $0.1-1 \mu \mathrm{mol} / \mathrm{L}$ & 2360 & Babendure et al., 2003 \\
GFP like fluorophores & $80 \mathrm{nt}$ & $0.5 \mu \mathrm{mol} / \mathrm{L}$ & 2000 & Paige et al., 2011 \\
Hoechst 1C & $29 \mathrm{nt}$ & $35 \mu \mathrm{mol} / \mathrm{L}$ & 56.2 & Sando et al., 2008 \\
Dimethyl indole red & $102 \mathrm{nt}$ & $86 \mu \mathrm{mol} / \mathrm{L}$ & 60 & Constantin et al., 2008 \\
ASR7 & $60 \mathrm{nt}$ & $2.6 \mu \mathrm{mol} / \mathrm{L}$ & 95 & Lee et al., 2010 \\
DCF-MPP & $110 \mathrm{nt}$ & $\mathrm{N} . \mathrm{D}$. & $13^{\text {a }}$ & Sparano and Koide, 2007 \\
Fluorophore-BHQ1 & $102 \mathrm{nt}$ & $4.7 \mu \mathrm{mol} / \mathrm{L}$ & $\sim 7$ & Murata et al., 2011 \\
\hline
\end{tabular}

a 13 -time increase at $100 \mu \mathrm{mol} / \mathrm{L}$ aptamer

N.D., not determined; DCF-MPP, 2',7'-dichlorofluorescein conjugated with two 1-(4-methoxyphenyl) piperazine

Some fluorogens cannot emit fluorescence due to intramolecular motions that cause excitation energy loss. However, when the intramolecular motion is restricted, the excitation energy can be utilized to emit fluorescence. Aptamers can be developed to bind such fluorogens to restrict their intramolecular motion, thus making the fluorogens fluoresce upon aptamer binding. Such aptamer-fluorogenic dye pairs are thus called light-up probes. The malachite green-aptamer pair (Babendure et al., 2003) and GFP like fluorophores-aptamers pairs (Paige et al., 2011) have been successfully developed with submicromolar affinity and about 2000 times increase in fluorescence quantum yield between free and aptamer-bound form of the dyes. Hoechst 1c-aptamer pair (Sando et al., 2008) and dimethyl indole red -aptamer pair (Constantin et al., 2008) are other examples in which the affinity of the aptamer-dye interaction is 35 and 86 $\mathrm{nmol} / \mathrm{L}$ respectively and the fluorescence enhancement of the dyes was exhibited by 56 and 60 folds respectively upon aptamer binding.

In addition, a fluorogen can be generated by conjugating a fluorescent dye to a quencher. Then an aptamer can be developed to either bind the whole dye (ASR7) (Lee et al., 2010) or just the quencher (MPP in DCF-MPP or BHQ1 in fluorophore-BHQ1) (Sparano and Koide, 2007; Murata et al., 2011), resulting in fluorescence enhancement of 7-100 times.

Although some fluorogen-aptamer pairs have been developed as mentioned above and have been used to measure and monitor transcription in test tube in real-time (Sando et al., 2008), only the spinach-DFHBI pair (one of the pairs in GFP like fluorophore-aptamer pairs) (Paige et al., 2011) has been successfully applied for imaging the highly abundant cellular RNA, 5S rRNA in cell culture (Fig. 3B). Among fluorogen-aptamer pairs, many of the aptamers have large size (60-110 nt long). To image low abundance cellular RNAs (e.g., mRNA), multiple units of an aptamer tag are often needed. Aptamers with smaller size are needed for tagging RNA with minimal interference. For small fluorogenic dyes, there are some issues relating to in vivo application too. For example, cytotoxicity and strong nucleic staining of malachite green (our unpublished data) limits its application for in vivo for in vivo RNA imaging. Future development of fluorogen-aptamer pairs is in need with high affinity and specificity, small aptamer size and fluorogens with good cell-permeability, no toxicity, low background binding/noise and strong fluorescence enhancement upon aptamer binding.

\section{RNA imaging based on hybridization-regulated allosteric aptamers}

In addition to aptamer tagging, hybridization-regulated allosteric aptamers have also been developed for RNA measurement in test tube. In this approach, regions accessible for hybridization on a target RNA need to be identified for probe design. In addition, hybridization may affect RNA behavior, e.g., stability. The advantage of this approach is that it can potentially be used to image and quantify endogenous RNAs.

A targeted reversibly attenuated probe (TRAP) (Fig. 2, probe 10 ) is an example of hybridization regulated allosteric aptamers. In the TRAP, an intervening antisense sequence is flanked by an aptamer and an attenuator. The aptamer and the attenuator are complementary to each other to form a hairpin like structure of TRAP in the absence of a target nucleic acid. In the presence of a target nucleic acid, the target nucleic acid hybridizes with the intervening antisense sequence in the TRAP, thus breaking the hairpin structure formed between the aptamer and the attenuator. The unhybridized aptamer is then available to bind its ligand, which signals the presence of the target nucleic acid. The TRAP has been demonstrated to detect target nucleic acids in test tube with single nucleotide precision (Cong and Nilsen-Hamilton, 2005). The aptamer in the TRAP can be replaced with ribozyme for signal amplification through enzymatic reaction too (Burke et al., 2002).

A binary probe (Fig. 2, probe 11) is another example of hybridization regulated allosteric aptamers. In the probe, a malachite green aptamer is split into two halves, each linked to a short nucleotide sequence. The two short nucleotide sequences are antisense oligonucleotides to a target nucleic acid. In the absence of the target nucleic acid, the binary probe cannot come together to form a stable structure of a 
functional malachite green aptamer. However, in the presence of a target nucleic acid, the hybridization of the antisense oligonucleotides on the binary probes to the target nucleic acid brings the two halves of the malachite green aptamer together to become a functional malachite green aptamer. Binding of malachite green to the functional malachite green aptamer thus signals the presence of the target nucleic acids (Kolpashchikov, 2005).

Although both TRAP and binary probes have only been implemented in the test tube, the principles developed have the potential for in vivo endogenous RNA imaging.

\section{Proteins}

Proteins are essential for cellular functions in all aspects. Imaging proteins in cellular context is important for understanding their functions. In addition, many proteins can serve as disease diagnosis markers. Here we discuss the application of aptamers in imaging protein localization and trafficking in a cellular context, in sensing extracellular signal proteins and in diagnostic imaging in animal models (Table S1).

\section{Protein localization and trafficking in cells by aptamer imaging}

Protein localization and trafficking have been extensively studied by fusing the target proteins with fluorescent proteins as reporters. However, this tagging approach images the fusion proteins instead of the endogenously expressed target protein. As an alternative approach, direct aptamer probes can be used to image endogenous proteins. Aptamers can be labeled with a single reporter (Fig. 2, probe 1) or engineered as smart probes to image the cellular localization or trafficking dynamics of proteins. For live cell imaging of an intracellular protein, HIV reverse transcriptase (HIV RT), an aptamer beacon (Fig. 2, probe 3) was designed. In the aptamer beacon, a short oligonucleotide was added to complement part of an HIV RT aptamer to form a stem-loop structure. A TAMRA/DABCY pair was then conjugated respectively to the $5^{\prime}$ and the $3^{\prime}$ end of the aptamer beacon. In the absence of HIV-RT, TAMRA was quenched by adjacent $D A B C Y L$ due to proximity in the stem-loop structure. In the presence of HIV-RT, binding of HIV-RT to its aptamer broke the stem-loop structure, thus making DABCYL far away from TAMRA, which then emitted fluorescence for HIV-RT imaging. The aptamer beacon was delivered into HIV-RT transiently transfected HeLa cells or HIV-RT stably integrated U1 cells by streptolysin $\mathrm{O}$ treatment for reversible permeabilization. The aptamer beacon was shown to image cytoplasmic HIV-RT in living cells, which was consistent with imaging with HIV RT-GFP fusion protein (Liang et al., 2011). In another example of imaging cellular distribution of $\mathrm{PrP}^{\mathrm{c}}$, a silver nanoparticle functionalized by a $\operatorname{PrP}^{c}$ aptamer was applied to show $\operatorname{PrP}^{\mathrm{C}}$ cellular distribution on cell membrane, endocytotic structure, lysosome and mitochondria by both light scattering microscopy and transmission electron microscopy (Chen et al., 2010). Direct imaging of protein colocalization (nucleolin and integrin $\alpha v \beta_{3}$ ) based on FRET has also been reported (Kang et al., 2009b). In addition to cellular localization, fluorescence labeled aptamers have been used to study protein trafficking including endocytosis of human protein kinase-7 (PTK7) from membrane into endosome (Xiao et al., 2008) and endocytosis of angiogenin into cytoplasm and nuclei as scattered spots (Li et al., 2008). Particularly, protein trafficking dynamics has been imaged and quantified in real-time in living cells by an aptamer probe. In the probe, an aptamer conjugated with biotin at one end and with/without a fluorophore at the other end was used to recognize the target protein. Then a streptavidin-conjugated nanoparticle (quantum dot, silver nanoparticle or gold nanoparticle) was recruited to the target protein through biotin-streptavidin interaction. The target protein was then imaged by single/dual colors and the protein dynamics was determined by single particle tracking. The system was applied to image two membrane proteins nucleolin and $\operatorname{PrP}^{\mathrm{c}}$ and the endocytosis dynamics of $\mathrm{PrP}^{\mathrm{C}}$ without changing its endocytosis behavior. $\operatorname{PrP}^{C}$ endocytosis was mediated through a clathrin-dependent pathway with three distinct movement phases. On the cell membrane, the $\mathrm{PrP}^{\mathrm{C}}$ showed slow membrane diffusion with a velocity of 0.1 $\mu \mathrm{m} / \mathrm{min}$. In the cytoplasm, $\operatorname{PrP}^{c}$ showed continuous and directional movement with a velocity of $0.6 \mu \mathrm{m} / \mathrm{min}$ for vesicle transport into the lysosome. A small portion of $\mathrm{PrP}^{\mathrm{C}}$ could escape from the lysosome and get into the Golgi and the endoplasmic reticulum where $\mathrm{PrP}^{\mathrm{c}}$ showed confined diffusion, which was nondirectional and discontinuous with a velocity of 0.2-0.6 $\mu \mathrm{m} / \mathrm{min}$ (Chen et al., 2012).

\section{Extracellular signaling protein sensed by aptamer imaging}

In addition to imaging protein localization and trafficking, aptamer probes have been used to sense extracellular signaling molecules, e.g., platelet-derived growth factor (PDGF). For real-time imaging and quantifying extracellular PDGF, two different aptamer probes were constructed based either on fluorescence quenching (Fig. 2, probe 2) or on FRET (Fig. 2, probe 5). In the aptamer probes, a PDGF DNA aptamer with additional sequence hybridized to a complementary short oligonucleotide biotinylated at the $5^{\prime}$ end. The aptamer probes were thus coupled to the cell surface though streptavidin, which bound the cell surface biotinylated by sulphonated biotinyl-N-hydroxy-succinimide (NHS-biotin). In the absence of PDGF, the PDGF aptamer probes were in a open conformation. Binding of PDGF to the aptamer probes resulted in a closed conformation of the PDGF aptamer probes, which brought the labeled dyes (FAM vs Dabcyl and Cy3 vs Cy5) close enough to generate either quenching or FRET signal. The PDGF aptamer probes could quantify PDGF concentra- 
tion added in cell culture medium and secreted by neighboring cells with single-cell resolution. The aptamer probe-coupled stem cells still retained their homing ability to bone marrow, indicating the feasibility for the cell-surface coupled aptamer sensors for in vivo tracking and quantification of extracellular signaling molecules (Zhao et al., 2011).

\section{Diagnostic imaging of protein markers by aptamers}

Many proteins can serve as disease makers. Aptamers against such protein markers have great potential for diagnostic imaging with many advantages over antibodies due to their small size, no immunogenicity and easy chemical synthesis and chemical modification. The first success of in vivo aptamer diagnostic imaging was on human neutrophil elastase, a marker of inflammation. An aptamer against human neutrophil elastase was labeled with ${ }^{99 \mathrm{~m}} \mathrm{Tc}$ and injected intravenously to image an inflammation model in rat by scintigraphy. The ${ }^{99 \mathrm{~m}} \mathrm{Tc}$-labeled aptamer could image inflammation with peak signal/noise ratio of $4.3 \pm 0.6$ at $2 \mathrm{~h}$, which was superior to $\lg G$ with peak signal/noise ratio of $3.1 \pm 0.1$ at $3 \mathrm{~h}$ (Charlton et al., 1997). Another successful example of aptamer diagnostic imaging was on tenascin- $\mathrm{C}$, a cancer marker for various solid tumors. Aptamer TTA1 against tenascin- $C$ was labeled with either ${ }^{99 \mathrm{~m}} \mathrm{Tc}$ or rhodamine red and applied to image various tumor bearing mouse models including breast cancer (MDA-MB-435) and glioblastoma (U251) by either scintigraphy or fluorescence microscopy. The rhodamine red-labeled TTA1 aptamer accumulated at the blood vessels of the tumor by $10 \mathrm{~min}$ and diffused through the tumor tissue in $3 \mathrm{~h}$. The ${ }^{99 \mathrm{~m}} \mathrm{Tc}$-labeled aptamer showed tumor imaging with high signal/noise ratio over time (50 at $3 \mathrm{~h}$, 180 at $16 \mathrm{~h}$ ) due to fast tumor penetration (6\% injected dose/g at $10 \mathrm{~min}$ ), fast blood clearance (half-life $<2 \mathrm{~min}$ in blood) and long tumor retention (>20 h) (Fig. 3E) (Hicke et al., 2006).

In addition to scintigraphy imaging, whole-body fluorescence microscopy has been applied for diagnostic aptamer imaging of cancers. To image Ramos B-cell lymphoma in a nude mice model, TD05 aptamer was labeled with Cy5 and injected intravenously to specifically recognize immunoglobin heavy mu chain, a marker for Ramos B-cell lymphoma. After the injection, the Cy5-TD05 aptamer was rapidly taken up by the mouse body in just 4 min and cleared away from the body in 2-3 $\mathrm{h}$ but retained at the tumor site and intestine up to $6 \mathrm{~h}$. The recognition of Cy5-TD05 to Ramos tumor was specific with an average signal/noise ratio of $\sim 70$ at $5 \mathrm{~h}$ (Shi et al., 2010). To image CCRF-CEM cancer in nude mice, an activatable probe (Fig. 2, probe 3 ) was constructed to recognize PTK7, a marker for CCRF-CEM cancer. The probe was labeled with Cy5 at the $5^{\prime}$ end and BHQ2 at the $3^{\prime}$ end. In the absence of PTK7, the probe formed a hairpin structure and Cy5 was quenched by the adjacent BHQ2. In the presence of PTK7, Cy5 emitted fluorescence due to conformational change of the probe upon PTK7 binding, which placed BHQ2 far away from Cy5. The probe was injected intravenously to image CCRF-CEM tumor model in nude mice (Fig. 3D). The activatable probe was superior to Cy5-labeled aptamer (the always-on probe) on sensitivity and rapidity of diagnosis. When applied to tumor mouse model, the activatable probe was rapidly taken up in mouse in $5 \mathrm{~min}$ and significantly enriched at tumor site as early as $15 \mathrm{~min}$ and retained for more than $2 \mathrm{~h}$ after probe injection. On the other hand, the free probes were essentially cleared from the nontumor sites at 1 h. Recognition of the activatable probe was specific to the CCRF-CEM tumor (Shi et al., 2011).

Ultrasound imaging has been widely used for diagnosis. However, the conventional contrast reagents used in ultrasound imaging are often nonspecific. A contrast reagent conjugated with an aptamer has the potential to achieve targeted ultrasound imaging. To achieve targeted ultrasound imaging, sgc8c aptamer against PTK7 was conjugated to nanobubbles through thiol-maleimide chemistry. The aptamer conjugated nanobubbles were successfully used to image CCRF-CEM cells, which highly express PTK7 in cell culture. The ultrasound imaging was consistent with optical imaging. This demonstrated the feasibility to use the aptamer for targeted ultrasound imaging (Wang et al., 2011).

Computed tomography (CT) can achieve diagnostic imaging with or without contrast reagents. With aptamer-conjugated contrast reagents, targeted CT imaging could potentially be achieved. As an initial effort for targeted CT imaging in cell culture system, a gold nanoparticle (the contrast reagent) was functionalized with a short oligonucleotide, which was hybridized to a complementary short oligonucleotide extended from a PSMA aptamer. The aptamer-gold nanoparticle $(5 \mathrm{nmol} / \mathrm{L})$ was applied to image PSMA positive LNCaP prostate cancer cell with four times higher CT contrast than PSMA negative PC3 cell line (Kim et al., 2010).

Magnetic resonance imaging (MRI) is another widely used technique in diagnostic imaging. Similarly, targeted MRI can be achieved through aptamer-conjugated contrast reagent. In fact, a PSMA aptamer conjugated with a superparamagnetic iron oxide nanoparticle has been successfully applied to image LNCaP xenograft mouse model (Yu et al., 2011). Another example is a nucleolin aptamer conjugated with a cobalt-ferrite nanoparticle for imaging rat glioma (Hwang do et al., 2010).

\section{PERSPECTIVE}

We have evidenced many successes in applying aptamer-based molecular imaging on various molecules including small molecules, nucleic acids and proteins. These studies have promoted our further understanding of fundamental biology and shown the potential for aptamer-based imaging in translational medicine including diagnostic imag- 
ing and image-guided therapy. However, most of the aptamer-based molecular imaging has been done in a cellular context with limited examples of animal studies. For clinical translation of aptamer-based molecular imaging, interdisciplinary efforts are required from biology, chemistry, engineering, pharmacy and clinical medicine. Rapid and high throughput methodologies are in need for selecting avid and specific aptamers for a broad range of target molecules for in vivo application. New engineering approaches and reporter-labeling chemistries are expected to improve aptamer probes for high signal/noise ratio in imaging. For imaging intracellular targets, efficient and noninvasive intracellular delivery methods of aptamer probes need development. Good pharmacokinetic properties of aptamer probes are key to their in vivo application, which can be modulated by different chemistries. Finally, trials of aptamer probes for imaging in preclinical setting (animal models) and clinical setting need scientists and medical practitioners to explore the combinations of aptamer probes with different imaging modalities. We expect the interdisciplinary effort will bring aptamer-based molecular imaging to new horizons.

For aptamer-based small molecule imaging, there are only a few successful examples so far. Many aptamers have been selected in vitro to bind a broad range of small molecules, indicating the potential for aptamer-based small molecule imaging. The challenge will lie in the ability to select highly specific and avid aptamers for individual small molecules for in vivo application. With the ability of an aptamer to form a coherent yet malleable structure in controlling the chemical reactivity of its target (e.g., a metabolite) (Wang et al., 2009), there is great potential in combining imaging with phenotypic and functional studies of metabolites in cellular metabolism.

For aptamer-based RNA imaging, current methods mostly rely on aptamer-fluorescent protein tagging systems to image mRNAs. The large size of the fluorescent proteins and the aptamer tags can potentially alter the target RNA behavior. Aptamer-small molecule tagging systems and nontagging systems could potentially be developed as new imaging approaches to overcome the limitations of currents methods. Other than mRNAs, aptamer-based RNA imaging technologies have the potential for studying a broader range of RNAs including noncoding RNAs on their biogenesis, cellular localization, trafficking and function.

For aptamer-based protein imaging, aptamers developed against different epitopes of a protein have potential in imaging protein dynamics, conformational changes and molecular interactions inside cells. Multiplex imaging of different proteins may help to elucidate the interaction network, developmental signature and the resulting phenotypes. Aptamer-based diagnostic imaging of proteins as disease markers and image-guided therapy will also be actively investigated. In addition, aptamer-based molecular imaging may be used to study stem-cell development and monitor stem cells in experimental therapeutics.

\section{ACKNOWLEDGEMENTS}

The U.S. Department of Energy, Office of Biological and Environmental Research through the Ames Laboratory supported TJW and JR. The Ames Laboratory is operated for the U.S. Department of Energy by lowa State University under Contract No. DE-AC02$07 \mathrm{CH} 11358$. The authors thank Professor Marit Nilsen-Hamilton, Professor Gustavo MacIntosh, Dr. Timothy Herdendorf and Dr. Kirthi Narayanaswamy for their critical reading of this manuscript and their constructive advice. We also thank the anonymous reviewers for their helpful comments.

\section{ABBREVIATIONS}

CFP, cyan fluorescent protein; CT, computed tomography; FRET, Förster resonance energy transfer; GFP, green fluorescent protein; $\mathrm{MRI}$, magnetic resonance imaging; PDGF, platelet-derived growth factor; PET, positron emission tomography; PSMA, prostate-specific membrane antigen; PTK7, protein tyrosine kinase 7; QD, quantum dot; RFP, red fluorescent protein; RT, reverse transcriptase; SELEX, Systematic Evolution of Ligands by EXponential enrichment; SPECT, single photon emission computed tomography; TRAP, targeted reversibly attenuated probe; YFP, yellow fluorescente protein

\section{REFERENCES}

Austin, R.J., Xia, T., Ren, J., Takahashi, T.T., and Roberts, R.W. (2002). Designed arginine-rich RNA-binding peptides with picomolar affinity. J Am Chem Soc 124, 10966-10967.

Babendure, J.R., Adams, S.R., and Tsien, R.Y. (2003). Aptamers switch on fluorescence of triphenylmethane dyes. J Am Chem Soc 125, 14716-14717.

Bagalkot, V., Zhang, L., Levy-Nissenbaum, E., Jon, S., Kantoff, P.W., Langer, R., and Farokhzad, O.C. (2007). Quantum dot-aptamer conjugates for synchronous cancer imaging, therapy, and sensing of drug delivery based on bi-fluorescence resonance energy transfer. Nano Lett 7, 3065-3070.

Bao, G., Rhee, W.J., and Tsourkas, A. (2009). Fluorescent probes for live-cell RNA detection. Annu Rev Biomed Eng 11, 25-47.

Bermejo, C., Ewald, J.C., Lanquar, V., Jones, A.M., and Frommer, W.B. (2011). In vivo biochemistry: quantifying ion and metabolite levels in individual cells or cultures of yeast. Biochem J 438, 1-10.

Bertrand, E., Chartrand, P., Schaefer, M., Shenoy, S.M., Singer, R.H., and Long, R.M. (1998). Localization of ASH1 mRNA particles in living yeast. Mol Cell 2, 437-445.

Bi, J., Tsai, N.P., Lin, Y.P., Loh, H.H., and Wei, L.N. (2006). Axonal mRNA transport and localized translational regulation of kappa-opioid receptor in primary neurons of dorsal root ganglia. Proc Natl Acad Sci U S A 103, 19919-19924.

Brengues, M., Teixeira, D., and Parker, R. (2005). Movement of eukaryotic mRNAs between polysomes and cytoplasmic processing bodies. Science 310, 486-489.

Brodsky, A.S., and Silver, P.A. (2000). Pre-mRNA processing factors are required for nuclear export. RNA 6, 1737-1749.

Brody, E.N., Gold, L., Lawn, R.M., Walker, J.J., and Zichi, D. (2010). High-content affinity-based proteomics: unlocking protein biomarker discovery. Expert Rev Mol Diagn 10, 1013-1022. 
Burke, D.H., Ozerova, N.D., and Nilsen-Hamilton, M. (2002). Allosteric hammerhead ribozyme TRAPs. Biochemistry 41 , 6588-6594.

Charlton, J., Sennello, J., and Smith, D. (1997). In vivo imaging of inflammation using an aptamer inhibitor of human neutrophil elastase. Chem Biol 4, 809-816.

Chen, K., and Chen, X. (2010). Design and development of molecular imaging probes. Curr Top Med Chem 10, 1227-1236.

Chen, L.Q., Xiao, S.J., Hu, P.P., Peng, L., Ma, J., Luo, L.F., Li, Y.F., and Huang, C.Z. (2012). Aptamer-mediated nanoparticle-based protein labeling platform for intracellular imaging and tracking endocytosis dynamics. Anal Chem 84, 3099-3110.

Chen, L.Q., Xiao, S.J., Peng, L., Wu, T., Ling, J., Li, Y.F., and Huang, C.Z. (2010). Aptamer-based silver nanoparticles used for intracellular protein imaging and single nanoparticle spectral analysis. J Phys Chem B 114, 3655-3659.

Chubb, J.R., Trcek, T., Shenoy, S.M., and Singer, R.H. (2006). Transcriptional pulsing of a developmental gene. Curr Biol 16, 1018-1025.

Cong, X., and Nilsen-Hamilton, M. (2005). Allosteric aptamers: targeted reversibly attenuated probes. Biochemistry 44, 7945-7954.

Constantin, T.P., Silva, G.L., Robertson, K.L., Hamilton, T.P., Fague, K., Waggoner, A.S., and Armitage, B.A. (2008). Synthesis of new fluorogenic cyanine dyes and incorporation into RNA fluoromodules. Org Lett 10, 1561-1564.

Daigle, N., and Ellenberg, J. (2007). LambdaN-GFP: an RNA reporter system for live-cell imaging. Nat Methods 4, 633-636.

Darzacq, X., Shav-Tal, Y., de Turris, V., Brody, Y., Shenoy, S.M., Phair, R.D., and Singer, R.H. (2007). In vivo dynamics of RNA polymerase II transcription. Nat Struct Mol Biol 14, 796-806.

Das, B., Das, S., and Sherman, F. (2006). Mutant LYS2 mRNAs retained and degraded in the nucleus of Saccharomyces cerevisiae. Proc Natl Acad Sci U S A 103, 10871-10876.

Dictenberg, J.B., Swanger, S.A., Antar, L.N., Singer, R.H., and Bassell, G.J. (2008). A direct role for FMRP in activity-dependent dendritic mRNA transport links filopodial-spine morphogenesis to fragile X syndrome. Dev Cell 14, 926-939.

Dynes, J.L., and Steward, O. (2007). Dynamics of bidirectional transport of Arc mRNA in neuronal dendrites. J Comp Neurol 500, 433-447.

Ellington, A.D., and Szostak, J.W. (1990). In vitro selection of RNA molecules that bind specific ligands. Nature 346, 818-822.

Fang, Y., and Spector, D.L. (2007). Identification of nuclear dicing bodies containing proteins for microRNA biogenesis in living Arabidopsis plants. Curr Biol 17, 818-823.

Fusco, D., Accornero, N., Lavoie, B., Shenoy, S.M., Blanchard, J.M., Singer, R.H., and Bertrand, E. (2003). Single mRNA molecules demonstrate probabilistic movement in living mammalian cells. Curr Biol 13, 161-167.

Hicke, B.J., Stephens, A.W., Gould, T., Chang, Y.F., Lynott, C.K., Heil, J., Borkowski, S., Hilger, C.S., Cook, G., Warren, S., et al. (2006). Tumor targeting by an aptamer. J Nucl Med 47, 668-678.

Hong, H., Goel, S., Zhang, Y., and Cai, W. (2011). Molecular imaging with nucleic acid aptamers. Curr Med Chem 18, 4195-4205.

Hwang do, W., Ko, H.Y., Lee, J.H., Kang, H., Ryu, S.H., Song, I.C., Lee, D.S., and Kim, S. (2010). A nucleolin-targeted multimodal nanoparticle imaging probe for tracking cancer cells using an aptamer. J Nucl Med 51, 98-105.

lliuk, A.B., Hu, L., and Tao, W.A. (2011). Aptamer in bioanalytical applications. Anal Chem 83, 4440-4452.

Itzkovitz, S., and van Oudenaarden, A. (2011). Validating transcripts with probes and imaging technology. Nat Methods 8, S12-19.

Janicki, S.M., Tsukamoto, T., Salghetti, S.E., Tansey, W.P., Sachidanandam, R., Prasanth, K.V., Ried, T., Shav-Tal, Y., Bertrand, E., Singer, R.H., et al. (2004). From silencing to gene expression: real-time analysis in single cells. Cell 116, 683-698.

Jhaveri, S., Rajendran, M., and Ellington, A.D. (2000). In vitro selection of signaling aptamers. Nat Biotechnol 18, 1293-1297.

Kang, W.J., Chae, J.R., Cho, Y.L., Lee, J.D., and Kim, S. (2009a). Multiplex imaging of single tumor cells using quantum-dot-conjugated aptamers. Small 5, 2519-2522.

Kang, W.J., Ko, M.H., Lee, D.S., and Kim, S. (2009b). Bioimaging of geographically adjacent proteins in a single cell by quantum dot-based fluorescent resonance energy transfer. Proteomics Clin Appl 3, 1383-1388.

Keefe, A.D., Pai, S., and Ellington, A. (2010). Aptamers as therapeutics. Nat Rev Drug Discov 9, 537-550.

Kim, D., Jeong, Y.Y., and Jon, S. (2010). A drug-loaded aptamer-gold nanoparticle bioconjugate for combined $\mathrm{CT}$ imaging and therapy of prostate cancer. ACS Nano 4, 3689-3696.

Knopp, D., Tang, D., and Niessner, R. (2009). Review: bioanalytical applications of biomolecule-functionalized nanometer-sized doped silica particles. Anal Chim Acta 647, 14-30.

Ko, M.H., Kim, S., Kang, W.J., Lee, J.H., Kang, H., Moon, S.H., Hwang do, W., Ko, H.Y., and Lee, D.S. (2009). In vitro derby imaging of cancer biomarkers using quantum dots. Small 5, 1207-1212.

Kolpashchikov, D.M. (2005). Binary malachite green aptamer for fluorescent detection of nucleic acids. J Am Chem Soc 127, 12442-12443.

Lange, S., Katayama, Y., Schmid, M., Burkacky, O., Brauchle, C., Lamb, D.C., and Jansen, R.P. (2008). Simultaneous transport of different localized mRNA species revealed by live-cell imaging. Traffic 9, 1256-1267.

Lee, J., Lee, K.H., Jeon, J., Dragulescu-Andrasi, A., Xiao, F., and Rao, J. (2010). Combining SELEX screening and rational design to develop light-up fluorophore-RNA aptamer pairs for RNA tagging. ACS Chem Biol 5, 1065-1074.

Li, W., Yang, X., Wang, K., Tan, W., He, Y., Guo, Q., Tang, H., and Liu, J. (2008). Real-time imaging of protein internalization using aptamer conjugates. Anal Chem 80, 5002-5008.

Liang, Y., Zhang, Z., Wei, H., Hu, Q., Deng, J., Guo, D., Cui, Z., and Zhang, X.E. (2011). Aptamer beacons for visualization of endogenous protein HIV-1 reverse transcriptase in living cells. Biosens Bioelectron 28, 270-276.

Lim, F., and Peabody, D.S. (1994). Mutations that increase the affinity of a translational repressor for RNA. Nucleic Acids Res 22, 3748-3752.

Lopez-Colon, D., Jimenez, E., You, M., Gulbakan, B., and Tan, W. (2011). Aptamers: turning the spotlight on cells. Wiley Interdiscip Rev Nanomed Nanobiotechnol 3, 328-340.

Massoud, T.F., and Gambhir, S.S. (2003). Molecular imaging in living subjects: seeing fundamental biological processes in a new light. 
Genes Dev 17, 545-580.

Muramoto, T., Cannon, D., Gierlinski, M., Corrigan, A., Barton, G.J., and Chubb, J.R. (2012). Live imaging of nascent RNA dynamics reveals distinct types of transcriptional pulse regulation. Proc Natl Acad Sci U S A 109, 7350-7355.

Murata, A., Sato, S., Kawazoe, Y., and Uesugi, M. (2011). Small-molecule fluorescent probes for specific RNA targets. Chem Commun (Camb) 47, 4712-4714.

Nevo-Dinur, K., Nussbaum-Shochat, A., Ben-Yehuda, S., and Amster-Choder, O. (2011). Translation-independent localization of mRNA in E. coli. Science 331, 1081-1084.

Ng, K., Daigle, N., Bancaud, A., Ohhata, T., Humphreys, P., Walker, R., Ellenberg, J., and Wutz, A. (2011). A system for imaging the regulatory noncoding Xist RNA in living mouse embryonic stem cells. Mol Biol Cell 22, 2634-2645.

Nielsen, L.J., Olsen, L.F., and Ozalp, V.C. (2010). Aptamers embedded in polyacrylamide nanoparticles: a tool for in vivo metabolite sensing. ACS Nano 4, 4361-4370.

Nutiu, R., and Li, Y. (2005). Aptamers with fluorescence-signaling properties. Methods 37, 16-25.

Oguro, A., Ohtsu, T., Svitkin, Y.V., Sonenberg, N., and Nakamura, Y. (2003). RNA aptamers to initiation factor 4A helicase hinder cap-dependent translation by blocking ATP hydrolysis. RNA 9, 394-407.

Ohtsuka, K., Sato, S., Sato, Y., Sota, K., Ohzawa, S., Matsuda, T., Takemoto, K., Takamune, N., Juskowiak, B., Nagai, T., et al. (2012). Fluorescence imaging of potassium ions in living cells using a fluorescent probe based on a thrombin binding aptamerpeptide conjugate. Chem Commun (Camb) 48, 4740-4742.

Ozalp, V.C., Nielsen, L.J., and Olsen, L.F. (2010a). An aptamer-based nanobiosensor for real-time measurements of ATP dynamics. Chembiochem 11, 2538-2541.

Ozalp, V.C., Pedersen, T.R., Nielsen, L.J., and Olsen, L.F. (2010b). Time-resolved measurements of intracellular ATP in the yeast Saccharomyces cerevisiae using a new type of nanobiosensor. J Biol Chem 285, 37579-37588.

Paige, J.S., Nguyen-Duc, T., Song, W., and Jaffrey, S.R. (2012). Fluorescence imaging of cellular metabolites with RNA. Science 335, 1194.

Paige, J.S., Wu, K.Y., and Jaffrey, S.R. (2011). RNA mimics of green fluorescent protein. Science 333, 642-646.

Palmer, A.E., Qin, Y., Park, J.G., and McCombs, J.E. (2011). Design and application of genetically encoded biosensors. Trends Biotechnol 29, 144-152.

Pysz, M.A., Gambhir, S.S., and Willmann, J.K. (2010). Molecular imaging: current status and emerging strategies. Clin Radiol 65, 500-516.

Rackham, O., and Brown, C.M. (2004). Visualization of RNA-protein interactions in living cells: FMRP and IMP1 interact on mRNAs. EMBO J 23, 3346-3355.

Rook, M.S., Lu, M., and Kosik, K.S. (2000). CaMKIlalpha 3' untranslated region-directed mRNA translocation in living neurons: visualization by GFP linkage. J Neurosci 20, 6385-6393.

Sando, S., Narita, A., Hayami, M., and Aoyama, Y. (2008). Transcription monitoring using fused RNA with a dye-binding light-up aptamer as a tag: a blue fluorescent RNA. Chem Commun (Camb), 3858-3860.
Schmidt, U., Basyuk, E., Robert, M.C., Yoshida, M., Villemin, J.P., Auboeuf, D., Aitken, S., and Bertrand, E. (2011). Real-time imaging of cotranscriptional splicing reveals a kinetic model that reduces noise: implications for alternative splicing regulation. $\mathrm{J}$ Cell Biol 193, 819-829.

Shav-Tal, Y., Darzacq, X., Shenoy, S.M., Fusco, D., Janicki, S.M., Spector, D.L., and Singer, R.H. (2004). Dynamics of single mRNPs in nuclei of living cells. Science 304, 1797-1800.

Shi, H., He, X., Wang, K., Wu, X., Ye, X., Guo, Q., Tan, W., Qing, Z., Yang, X., and Zhou, B. (2011). Activatable aptamer probe for contrast-enhanced in vivo cancer imaging based on cell membrane protein-triggered conformation alteration. Proc Natl Acad Sci U S A 108, 3900-3905.

Shi, H., Tang, Z., Kim, Y., Nie, H., Huang, Y.F., He, X., Deng, K., Wang, K., and Tan, W. (2010). In vivo fluorescence imaging of tumors using molecular aptamers generated by cell-SELEX. Chem Asian J 5, 2209-2213.

Shui, B., Ozer, A., Zipfel, W., Sahu, N., Singh, A., Lis, J.T., Shi, H., and Kotlikoff, M.I. (2012). RNA aptamers that functionally interact with green fluorescent protein and its derivatives. Nucleic Acids Res 40, e39.

Smolander, O.P., Kandhavelu, M., Mannerstrom, H., Lihavainen, E., Kalaichelvan, S., Healy, S., Yli-Harja, O., Karp, M., and Ribeiro, A.S. (2011). Cell-to-cell diversity in protein levels of a gene driven by a tetracycline inducible promoter. BMC Mol Biol 12, 21.

Sparano, B.A., and Koide, K. (2007). Fluorescent sensors for specific RNA: a general paradigm using chemistry and combinatorial biology. J Am Chem Soc 129, 4785-4794.

Stojanovic, M.N., and Kolpashchikov, D.M. (2004). Modular aptameric sensors. J Am Chem Soc 126, 9266-9270.

Suhling, K., French, P.M., and Phillips, D. (2005). Time-resolved fluorescence microscopy. Photochem Photobiol Sci 4, 13-22.

Toomre, D., and Bewersdorf, J. (2010). A new wave of cellular imaging. Annu Rev Cell Dev Biol 26, 285-314.

Tuerk, C., and Gold, L. (1990). Systematic evolution of ligands by exponential enrichment: RNA ligands to bacteriophage T4 DNA polymerase. Science $249,505-510$.

Valencia-Burton, M., McCullough, R.M., Cantor, C.R., and Broude, N.E. (2007). RNA visualization in live bacterial cells using fluorescent protein complementation. Nat Methods 4, 421-427.

Valencia-Burton, M., Shah, A., Sutin, J., Borogovac, A., McCullough, R.M., Cantor, C.R., Meller, A., and Broude, N.E. (2009). Spatiotemporal patterns and transcription kinetics of induced RNA in single bacterial cells. Proc Natl Acad Sci U S A 106, 16399-16404.

Wang, C.H., Huang, Y.F., and Yeh, C.K. (2011). Aptamer-conjugated nanobubbles for targeted ultrasound molecular imaging. Langmuir 27, 6971-6976.

Wang, T. (2008). Function and dynamics of aptamers: A case study on malachite green aptamer. Ph.D.dissertation, lowa State University. ProQuest/UMI access number: AAT 3342297.

Wang, T., Hoy, J.A., Lamm, M.H., and Nilsen-Hamilton, M. (2009). Computational and experimental analyses converge to reveal a coherent yet malleable aptamer structure that controls chemical reactivity. J Am Chem Soc 131, 14747-14755.

Wang, Y., Li, Z., Hu, D., Lin, C.T., Li, J., and Lin, Y. (2010). Aptamer/graphene oxide nanocomplex for in situ molecular 
probing in living cells. J Am Chem Soc 132, 9274-9276.

Weil, T.T., Forrest, K.M., and Gavis, E.R. (2006). Localization of bicoid mRNA in late oocytes is maintained by continual active transport. Dev Cell 11, 251-262.

Weissleder, R., and Pittet, M.J. (2008). Imaging in the era of molecular oncology. Nature 452, 580-589.

Xiao, Z., Shangguan, D., Cao, Z., Fang, X., and Tan, W. (2008). Cell-specific internalization study of an aptamer from whole cell selection. Chemistry 14, 1769-1775.

Yamagishi, M., Ishihama, Y., Shirasaki, Y., Kurama, H., and Funatsu, T. (2009). Single-molecule imaging of beta-actin mRNAs in the cytoplasm of a living cell. Exp Cell Res 315, 1142-1147.

Yang, L., Zhang, X., Ye, M., Jiang, J., Yang, R., Fu, T., Chen, Y., Wang, K., Liu, C., and Tan, W. (2011). Aptamer-conjugated nanomaterials and their applications. Adv Drug Deliv Rev 63, 1361-1370.

Yiu, H.-W., Demidov, V.V., Toran, P., Cantor, C.R., and Broude, N.E. (2011). RNA Detection in live bacterial cells using fluorescent protein complementation triggered by interaction of two RNA aptamers with two RNA-binding peptides. Pharmaceuticals 4, 494-508.
Yu, M.K., Kim, D., Lee, I.H., So, J.S., Jeong, Y.Y., and Jon, S. (2011). Image-guided prostate cancer therapy using aptamer-functionalized thermally cross-linked superparamagnetic iron oxide nanoparticles. Small 7, 2241-2249.

Zeng, Q., and Hall, K.B. (1997). Contribution of the C-terminal tail of U1A RBD1 to RNA recognition and protein stability. RNA 3, 303-314.

Zhao, W., Schafer, S., Choi, J., Yamanaka, Y.J., Lombardi, M.L., Bose, S., Carlson, A.L., Phillips, J.A., Teo, W., Droujinine, I.A., et al. (2011). Cell-surface sensors for real-time probing of cellular environments. Nat Nanotechnol 6, 524-531.

Zheng, D., Seferos, D.S., Giljohann, D.A., Patel, P.C., and Mirkin, C.A. (2009). Aptamer nano-flares for molecular detection in living cells. Nano Lett 9, 3258-3261.

Zhou, J., Battig, M.R., and Wang, Y. (2010). Aptamer-based molecular recognition for biosensor development. Anal Bioanal Chem 398, 2471-2480.

Zimyanin, V.L., Belaya, K., Pecreaux, J., Gilchrist, M.J., Clark, A., Davis, I., and St Johnston, D. (2008). In vivo imaging of oskar mRNA transport reveals the mechanism of posterior localization. Cell 134, 843-853. 